\title{
Nonlinear transfer and temporal gain control in ON bipolar cells
}

\author{
Nikhil R. Deshmukh ${ }^{1}$ and Michael J. Berry II $^{1}$ \\ ${ }^{1}$ Princeton Neuroscience Institute, Princeton University, Princeton, New Jersey 08544
}

\begin{abstract}
The separation of visual input into discrete channels begins at the photoreceptor to bipolar cell synapse. Current models of the ON pathway describe the time-varying membrane voltage of $\mathrm{ON}$ bipolar cells as a linear function of light fluctuations. While this linearity holds under some visual conditions, stimulating the retina with full-field, high contrast flashes reveals a number of nonlinearities already present in the input current of ON bipolar cells. First, we show that the synaptic input to ON bipolar cells is asymmetric in response to equal flashes of opposite polarity. Next, we show that this asymmetry emerges because the responses to dark flashes increase linearly with contrast, whereas responses to bright flashes are highly rectified. We also describe how the outward current saturates in response to dark flashes of increasing duration. Furthermore, varying the inter-flash interval between a pair of high contrast flashes reveals a rapid, transient form of gain control that modulates both the amplitude and time course of the flash response. We develop a phenomenological model that captures the primary features of the ON bipolar cell response at high contrast. Finally, we discuss the implications of these nonlinearities in our understanding of how retinal circuitry shapes the visual signal.
\end{abstract}

\section{Introduction}

Bipolar cells, the second-order neurons of the retina, are increasingly understood to be the site of important and powerful visual computations [1]. Their axon terminals can convert membrane voltage into a graded release of glutamate in a manner embodying rectification of the visual signal. As a result of this rectification, bipolar cells act as nonlinear subunits within a ganglion cell's receptive field [2-5], thereby giving rise to forms of translation invariance in retinal motion processing [6-8]. Furthermore, synaptic depression within the axon terminal can dramatically reduce glutamate release [9-11], giving rise to a form of gain control that emphasizes motion discontinuities $[8,12]$.

In addition to these image transformations at its output, bipolar cells can also carry out powerful computations in their dendrites through the action of their glutamate receptors. In order to more efficiently encode increments and decrements of light intensity, the retina splits the visual signal into parallel ON and OFF pathways [13]. OFF bipolar cells have ionotropic receptors that preserve the (inverted) sign of visual signals, and both kainateand AMPA-type receptors exhibit a strong form of desensitization with different kinetics $[14,15]$. ON bipolars have a metabotropic receptor, mGluR6, that inverts the sign of their glutamate signals to produce depolarizing responses to increases in light intensity [16]. Activation of mGluR6 by binding of glutamate triggers a second messenger cascade that 
ultimately closes the cation channel TRPM1 [17-20], although the full biochemical pathway has not been delineated. Because ON bipolars use this signaling cascade, they have the potential to carry out even more sophisticated computations within their dendrites than OFF bipolars.

Indeed, at low light level rod bipolar cells have a threshold nonlinearity that helps improve signal-to-noise ratio for the integration of single-photon events generated by rod photoreceptors [21]. This nonlinearity results from saturation in the mGluR6 cascade [22]. At higher light levels, ON bipolar cells exhibit saturation for both positive and negative contrasts [23, 24]. The mGluR6 cascade exhibits desensitization via feedback from intracellular calcium on a timescale of $\sim 0.8 \mathrm{sec}$ [25] as well as potentiation via cGMP [26]. At shorter timescales, however, the dynamics of the cascade are not as well characterized. Another approach to studying the transformation of light to ON bipolar cell activity has ignored this biophysical complexity. Instead, the response characteristics were elucidated using reverse correlation to spatiotemporal white noise stimulation. Under these conditions, most of the fluctuations in membrane voltage were captured by a linear model [27-30] with bandpass temporal kernels [28, 31]. This kernel can adapt as a function of the mean membrane voltage due to the action of voltage-gated $\mathrm{K}+$ channels [32].

We seek to reconcile these different views and produce a unified model of ON bipolar cell function at the level of its synaptic input current. Our approach has been to study the synaptic currents into $\mathrm{ON}$ bipolar cells using voltage clamp measurements in the retinal slice preparation. We varied the contrast and duration of bright and dark flashes of light to probe both the linear and saturating regimes. We find that the synaptic input to ON bipolar cells already contains multiple sources of significant nonlinearity that are present for moderate contrast and short duration flashes of light. There are two sources of saturation giving rise to responses that are asymmetric for bright versus dark flashes. Input currents have a biphasic temporal kernel in which the overshoot increases strongly as a function of contrast. In addition, we found a gain control mechanism acting on the same time scale as the immediate light response. We describe a unified computational model that captures these nonlinearities in the ON bipolar cell light response. This model includes saturation at the photoreceptor-to-bipolar cell synapse, temporal gain control within the mGluR6 cascade, and saturation in the opening of the TRPM1 channel.

\section{Materials and Methods}

\section{Preparation}

Experiments were performed on larval tiger salamanders (Charles Sullivan, Nashville, TN) kept at $16^{\circ} \mathrm{C}$ on a 12 -hr light-dark cycle. Care and euthanasia of the animals were carried out in accordance with procedures approved by Princeton University Animal Care and Use Committee. After being dark-adapted for 2 hours, salamanders were rapidly decapitated and the head and spinal cord pithed under dim illumination; all subsequent steps were performed under infrared illumination. The eyes were removed and hemisected, and the cornea, iris, lens, and vitreous humor were removed. The retina was embedded in low melting temperature agarose (Sigma) made with HEPES-buffered AMES medium and sliced into $250 \mathrm{um}$ thick slices on a vibrating microtome (Leica) at $4^{\circ} \mathrm{C}$.

\section{Electrophysiology}

All recordings were made at room temperature in bicarbonate-buffered Ringers solution containing (in $\mathrm{mM}$ ): $110 \mathrm{NaCl}, 22 \mathrm{NaHCO}_{3}, 2.5 \mathrm{KCl}, 1.6 \mathrm{MgCl}_{2}, 1 \mathrm{CaCl}_{2}$, and 10 glucose, 
equilibrated with $95 \% \mathrm{O}_{2}, 5 \% \mathrm{CO}_{2}$, and adjusted to $\mathrm{pH} 7.35$ with $\mathrm{NaHCO}_{3}$. Retinae were viewed under infrared illumination, and individual bipolar cells were patched using standard whole-cell patch techniques. Patch electrodes were pulled from $1.2 \mathrm{~mm} O \mathrm{OD}$ borosilicate glass (FHC) on a Sutter P-2000 micropipette puller. Electrode diameters were approximately $1.0 \mu \mathrm{m}$ at the tip, and had resistances of 8-12 $\mathrm{M} \Omega$. All whole-cell patch recordings were performed with a Multiclamp 700B patch-clamp amplifier (Molecular Devices). Bipolar cell intracellular solution contained (in $\mathrm{mM}$ ): $125 \mathrm{~K}$-aspartate, $10 \mathrm{KCl}$, $10 \mathrm{HEPES}, 5$ EDTA, $1 \mathrm{CaCl}_{2}, 1 \mathrm{ATP}$, and $0.5 \mathrm{GTP}$ adjusted to $\mathrm{pH} 7.3$ with NMG-OH. Bipolar cell types were confirmed by measuring light responses, and cells with a series resistance $>50 \mathrm{M} \Omega$ were discarded. The liquid junction potential $(-9 \mathrm{mV})$ was uncorrected.

\section{Visual stimulation}

Full field flash stimuli were delivered by a $670 \mathrm{~nm}$ LED (SuperBrightLEDS) behind a holographic diffuser (Thorlabs) focused onto the retina from the bottom of the recording chamber. Bright and dark flashes of high contrast ( \pm 1.0 Weber contrast) and varying duration were interleaved and presented on background illumination to define the adaptational state of the retina in the photopic regime. We presented a set of 50 random binary white noise sequences, each having a duration of 5 seconds, a bandwidth of $30 \mathrm{~Hz}$, and a temporal contrast of 33\% (standard deviation across time divided by the mean). Of these sequences, 15 were identical and served as the test sequence for the linear model, and the remaining 35 were unique and were used to calculate the parameters of a linearnonlinear model. The mean background light intensity of all stimuli measured at the plane of the retina was 1650 photons/L-cone/sec. Photon flux was calculated using salamander photoreceptor spectra values reported in the literature [33, 34]. The retina was continuously illuminated by a rod-suppressing $540 \mathrm{~nm}$ green background to isolate cone circuitry.

\section{Analysis}

Data were acquired at a sampling rate of $20 \mathrm{kHz}$ and low-passed filtered at $1 \mathrm{kHz}$ using pClamp software (Axon Instruments, Foster City, CA). Subsequent analysis was performed using SciPy, an open-source scientific computing environment for the Python programming language. The linear filter in Figure 3A was calculated as in [35]. Following [36], the nonlinearity (Figure 3B and 4A) was determined by binning the predicted response into $0.5 \mathrm{mV}$ bins and calculating the average measured current response for each bin.

\section{Computational Model}

We developed a Linear-Nonlinear-Kinetic-Nonlinear (LNKN) model to predict the synaptic input current to $\mathrm{ON}$ bipolar cells over the entire range of visual stimuli that we tested experimentally. The stimulus $s(t)$ is the Weber contrast of the full-field luminance $Y$ as a function of time:

$$
s(t)=\frac{Y(t)-Y_{b}}{Y_{b}}
$$

where $Y_{b}$ is the background luminance.

We modeled the photoreceptor voltage $L(t)$ as the convolution of the stimulus $s(t)$ with a linear kernel $k(t)$ as follows: 
where

$$
L(t)=-\int_{-\infty}^{\infty} d t^{\prime} k\left(t-t^{\prime}\right) s\left(t^{\prime}\right)
$$

$$
k(t)=\frac{1}{\Gamma(n+1)}\left(\frac{t}{\tau}\right)^{n} e^{-\frac{t}{\tau}}
$$

The negative sign represents the sign-inversion that produces a hyperpolarization in response to a positive contrast flash. The glutamate release at the photoreceptor $u(t)$ was given by the nonlinear function, $N_{1}$ :

$$
u(t)=N_{1}[L(t)]=\phi_{0}-\frac{\alpha_{1} L(t)}{1-\alpha_{1} L(t)}
$$

where $\phi_{0}$ represents the level of steady-state glutamate release at the background light level $(L=0)$, and $\alpha_{1}$ determines the slope of the input-output function. The units of glutamate release are truncated to the (normalized) range $[0,1]$.

The output of the photoreceptor, $u(t)$, is then passed through the kinetic block of the model, similar to [37]. This block captures the dynamics of the mGluR6 receptor to TRP channel current system by reducing all of the response elements and second messengers to three internal state variables: $R$ (ready pool of signaling elements), $A$ (active pool), and $X$ (inactive pool). The time evolution of these internal variables is given by a Markov process:

$$
\frac{d \boldsymbol{P}^{T}}{d t}=\boldsymbol{P}^{T}(t) \boldsymbol{Q}(u)
$$

where the fractional occupancies $R, A$, and I each make up a row in the column vector $\mathbf{P}(t)$ and satisfy the condition $R+A+I=1$. $\mathbf{Q}$ is a $3 \times 3$ transition matrix that determines the transitions between each of three states:

$$
\frac{d \boldsymbol{P}^{T}}{d t}=\left(\begin{array}{c}
R(t) \\
\cdot A(t) \\
\cdot \\
X(t)
\end{array}\right)=\boldsymbol{P}^{T}(t)\left(\begin{array}{ccc}
-u(t) k_{A} & u(t) k_{A} & 0 \\
0 & -k_{X} & k_{X} \\
k_{R} & 0 & -k_{R}
\end{array}\right)
$$

Glutamate release $u(t)$ scales the rate at which a signaling element in the $R$ pool transitions to the $A$ pool. The solution to this system of differential equations was obtained numerically. To determine the output of the kinetic block $c(t)$, we multiplied the occupancy of the active pool $A(t)$ by -1 to capture the sign-inversion where the channel closes when activated and subtracted the initial value at $t=0, A(0)$

$$
c(t)=-A(t)+A(0)
$$

Next, to convert $c(t)$ to an input current $I(t)$, we applied a static nonlinearity $N_{2}(t)$ and scaled the response to units of $p A$.

$$
I(t)=N_{2}(c(t))
$$


where $N$ is given by:

$$
N_{2}(t)=\left\{\begin{array}{c}
\beta c(t), A<0 \\
\frac{\beta}{1+\alpha_{2} c(t)^{2}}, A \geq 0
\end{array}\right.
$$

The term $\beta$ is the scale factor from arbitrary units to $\mathrm{pA}$, and $\alpha_{2}$ sets the slope of the rectification function.

The best fit parameters were determined using stochastic gradient descent, and are as follows:

\begin{tabular}{|c|l|l|}
\hline Parameters & Description & Cell 3011 \\
\hline$n$ & Exponent of linear photoreceptor kernel & 5.51 \\
\hline$\tau$ & Time constant of linear photoreceptor kernel & $10.1 \mathrm{~ms}$ \\
\hline$\phi_{0}$ & Constitutive glutamate release & 1.1 \\
\hline$\alpha_{1}$ & Slope of photoreceptor output nonlinearity & 0.52 \\
\hline$k_{R}$ & Transition rate, $\mathrm{X} \rightarrow \mathrm{R}$ & $0.0015 / \mathrm{ms}$ \\
\hline$k_{A}$ & Transition rate, $\mathrm{R} \rightarrow \mathrm{A}$ & $0.010 / \mathrm{ms}$ \\
\hline$k_{X}$ & Transition rate, $\mathrm{A} \rightarrow \mathrm{X}$ & $0.011 / \mathrm{ms}$ \\
\hline$\alpha_{2}$ & Slope, saturating nonlinearity & 10.5 \\
\hline$\beta$ & Scale factor, arbitrary units to $\mathrm{pA}$ & $31.8 \mathrm{pA}$ \\
\hline
\end{tabular}

\section{Results}

Linear superposition is a powerful and important mathematical property. When it holds, the complete response of a system can be derived from well-developed mathematical methods. On the other hand, linear systems embody limited computational powers, as the input can be recomputed from the output (assuming no noise is added). Thus, a nonlinear system, while more complicated to characterize, can be said to perform more substantial processing on its inputs. To directly test the linearity of the synaptic input to ON bipolar cells, we performed voltage-clamp recordings of ON bipolar cells in a slice preparation of the tiger salamander retina in response to full-field bright and dark flashes (see Materials and Methods). ON bipolar cells were identified by their morphology and depolarizing response to bright flashes, and were clamped at their resting membrane potential as measured in current clamp (mean \pm SEM $=-41.2 \pm 1.4 \mathrm{mV}, \mathrm{n}=12$ ).

We tested whether the synaptic input to these cells was linear at high contrast by comparing the amplitudes of the peak postsynaptic current in response to $50 \mathrm{~ms}$ full field 
flashes of \pm 1.0 Weber contrast. To facilitate this comparison visually, the mean dark flash response (blue trace) was inverted and superimposed on the mean bright flash response (red trace), as shown in Figure 1. The response to a strong flash is biphasic, with a sharp rise to peak and a subsequent overshoot in the opposite direction. In all ON bipolar cells recorded, the amplitude of the peak outward current and the peak inward overshoot in response to a dark flash was greater than the amplitudes of the corresponding peak and overshoot in response to a bright flash.

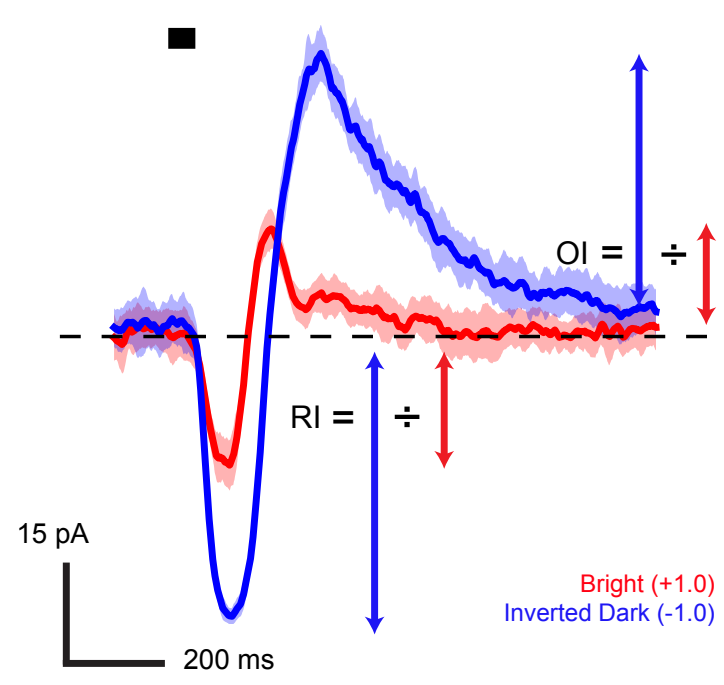

Figure 1. Comparison of the response to bright and dark flashes. Measured input current for an example ON bipolar current in response to $100 \%$ contrast bright (red trace) and dark flashes (blue trace). The black bar represents the duration of the $50 \mathrm{~ms}$ full-field stimulus. The solid traces represent the mean response and the light shaded regions around the traces show the standard deviation of the responses across 5 trials. The rectification index $(\mathrm{RI})$ and the overshoot index (OI) were used to quantify response asymmetry, as illustrated.

To quantify this asymmetry between ON and OFF responses, we defined the Rectification Index $(\mathrm{RI})$ of the cell as the ratio of the dark flash response amplitude to the maximal bright flash response amplitude. Analogously, we defined the Overshoot Index (OI) as the ratio of the overshoot in response to dark and bright flashes. For our population of cells, the mean RI was $2.70 \pm 0.41$ and the mean OI was $2.46 \pm 0.31$ (mean \pm SEM, $n=12$ ), indicating significant rectification of light increments compared to decrements at the cone to ON bipolar synapse (RI: $p=0.008$; OI: $p=0.015$, paired t-test).

To test whether this asymmetry persists at lower contrasts, we measured the rectification of the peak and overshoot in response to flashes of \pm 0.25 and \pm 0.5 Weber contrast. The maximal amplitude of the dark flash peak response and overshoot increased linearly as a function of contrast, whereas the corresponding peak and overshoot in response to a bright flash saturated at high contrast. This led to the increase of the mean rectification index from approximately 1 (symmetric) at 25\% contrast to greater than 2.5 (highly asymmetric) at $100 \%$ contrast. The emergence of this asymmetry as a function of contrast is shown for an example cell in Figure 2A. The saturation of the inward current in response to bright flashes at $50 \%$ contrast suggests that a signaling element within the ON bipolar pathway is fully activated such that a stronger stimulus cannot activate it further. 

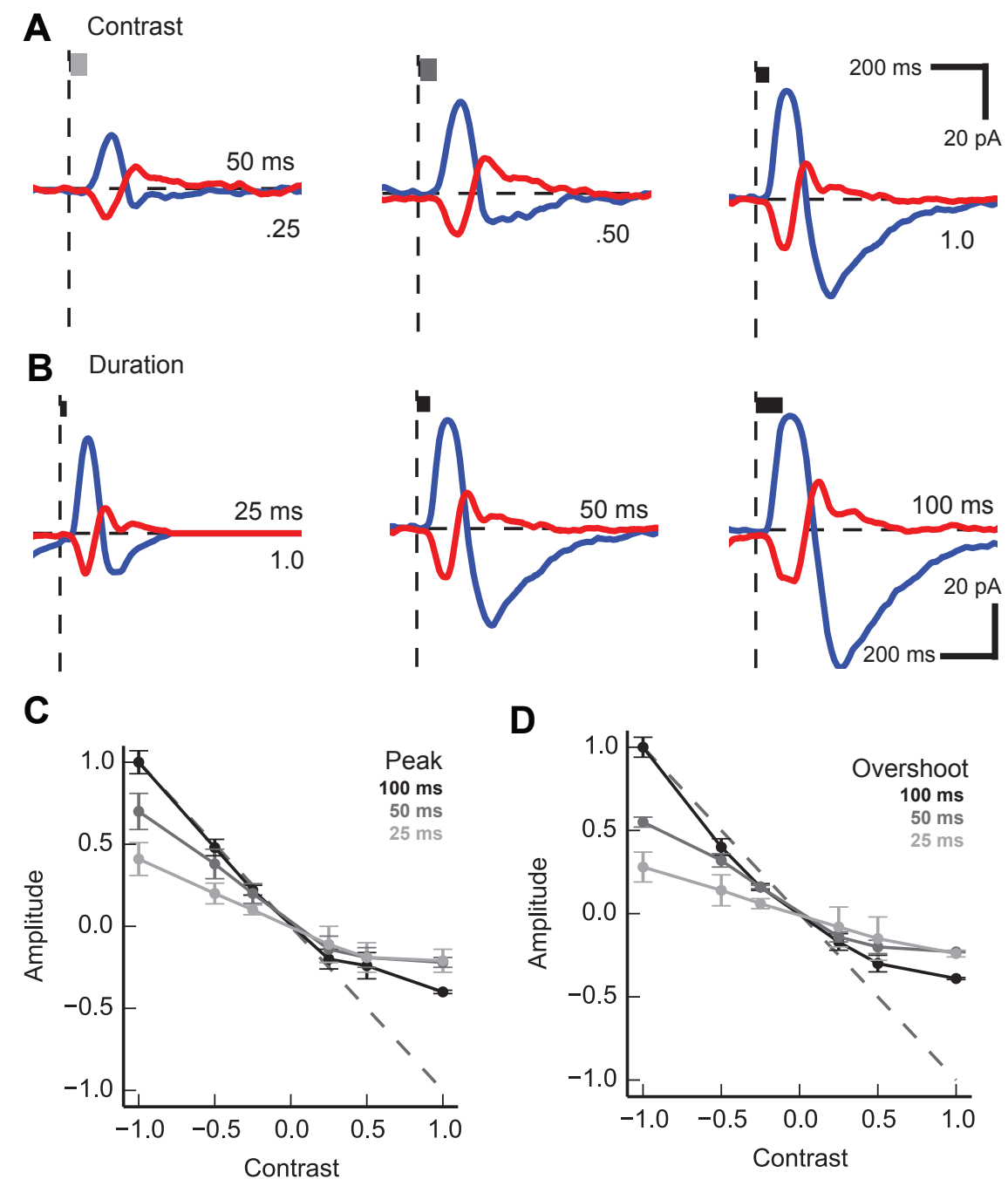

Figure 2. Response asymmetry between bright and dark flashes increases with contrast and flash duration A. ON bipolar postsynaptic current in response to bright and dark flashes of $25 \%$, $50 \%$ and $100 \%$ contrast. Traces represent mean response across 5 trials. B. ON bipolar postsynaptic current in response to flashes of 25,50 and $100 \mathrm{~ms}$ duration. Traces represent mean response across 5 trials. C. Peak response amplitude at all contrasts and durations normalized by the peak response at $100 \%$ contrast and $100 \mathrm{~ms}$ duration. Dotted line indicates unity and error bars represent SEM $(n=7)$. D. Peak overshoot amplitude at all contrasts and durations normalized by the peak overshoot at $100 \%$ contrast and $100 \mathrm{~ms}$ duration. Dotted line indicates unity and error bars represent SEM $(n=7)$.

Next, we examined the effect of changing the flash duration on the amplitude of the peak and overshoot. This is shown for an example cell in Figure 2B. The inward current in response to a bright flash saturated as the duration of the flash increased from 25 to $100 \mathrm{~ms}$. Similarly, the peak outward current in response to a dark flash also saturated over this range of flash durations. This suggests that sufficiently strong activation to produce rectification in the ON bipolar pathway can occur through temporal integration. 
Figure $2 \mathrm{C}$ shows the contrast response curve of the peak amplitude for all ON bipolar cells recorded. The amplitude was normalized to the peak response at the highest contrast. The saturation of the $\mathrm{ON}$ response required flashes of sufficient contrast and duration. For $50 \mathrm{~ms}$ and $100 \mathrm{~ms}$ flashes, the slope of the response amplitude as a function of contrast was significantly shallower at positive contrasts compared to negative contrasts. At $25 \mathrm{~ms}$, however, the slope was approximately constant from -1.0 to +0.5 contrast, with attenuation only at the +1.0 contrast condition.

The overshoot response normalized by the maximal overshoot (at high contrast and long duration) is shown in Figure 2D. The amplitude of the overshoot followed the same overall trend as the peak amplitude for all contrasts and durations. At low contrast and short duration, the overshoot was equally small for bright and dark flashes (compare contrasts \pm 1.0 ), consistent with a lack of rectification in this limit. However, as the flash duration was increased to 50 and $100 \mathrm{~ms}$, the overshoot in response to dark flashes grew significantly larger than that for bright flashes. The similarity of the overshoot contrast response to the peak contrast response suggests that the overshoot is a consequence of the saturation of the ON bipolar pathway.

Importantly, the peak outward current in response to dark flashes shown in Figure 2B does not increase with duration of the flash. In systems theory, the principle of linear superposition states that for all linear systems, the total response produced by the sum of many stimuli presented simultaneously is equal to the sum of the individual response of the system to each stimulus alone. Testing this superposition principle provides another method to probe the linearity of bipolar cell inputs. In Figure 3, we directly compared the OFF response of a single 50 or $100 \mathrm{~ms}$ flash to the superposition model, in which we summed either two or four time-shifted copies of the OFF response to a $25 \mathrm{~ms}$ flash. For the example cell shown, the peak amplitudes of the $50 \mathrm{~ms}$ and $100 \mathrm{~ms}$ superposition model were $60 \mathrm{pA}$ and $116 \mathrm{pA}$, respectively, but the measured peak outward current to either a $50 \mathrm{~ms}$ and $100 \mathrm{~ms}$ flash never exceeded $52 \mathrm{pA}$ (Figure 3A). Furthermore, the superposition model does not predict the large overshoots measured in response to 50 $\mathrm{ms}$ and $100 \mathrm{~ms}$ flashes.

To examine whether the rectification of the outward current depended on contrast, we tested for linearity using the 100 ms flash superposition model at $50 \%$ and $25 \%$ contrast. For each value of the superposition model, we averaged the corresponding values of the measured response. We then normalized all the responses by the peak outward current of the measured response at $100 \%$ contrast. As shown in Figure 3B, the normalized response at $100 \%$ contrast saturates quite strongly, with the peak amplitude of the superposition model reaching more than twice the peak amplitude of the measured data. At $50 \%$ contrast, the response still saturates, but the ratio of peak amplitudes is only 1.1 for the example cell shown. At $25 \%$ contrast, the measured response equal to the 
superposition model, indicating linearity. Figure 3C shows the mean ratio of peak amplitudes for the $50 \mathrm{~ms}$ and $100 \mathrm{~ms}$ superposition models and measured responses for all ON bipolar cells measured. Even for the shorter $50 \mathrm{~ms}$ flashes, the superposition model overpredicts the actual outward current measured. This suggests that there is a limit to the maximal outward current that an ON bipolar cell can sustain, and that the cell reaches this limit in response to high contrast dark flashes. At lower contrasts, this limit is not fully reached, so the response is more linear.

A
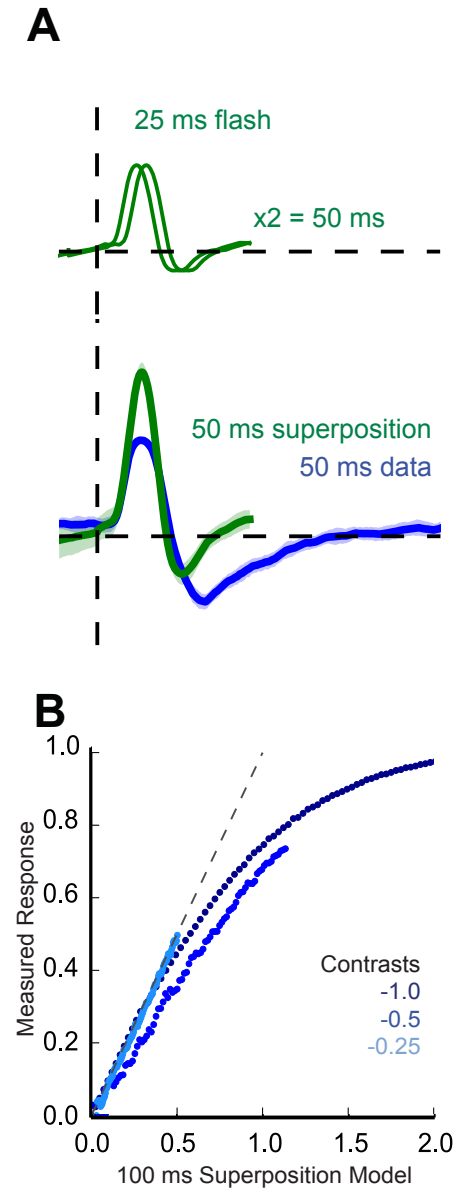

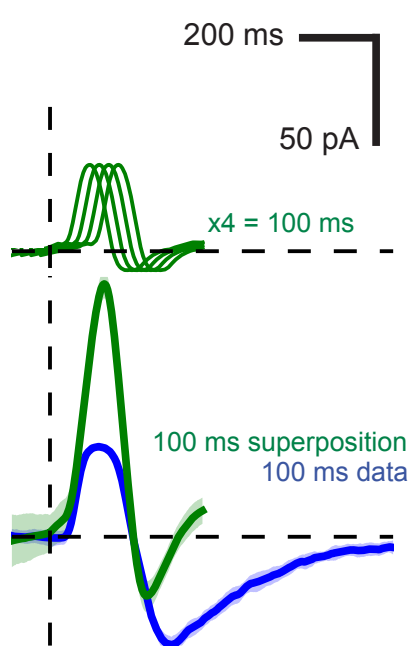

C

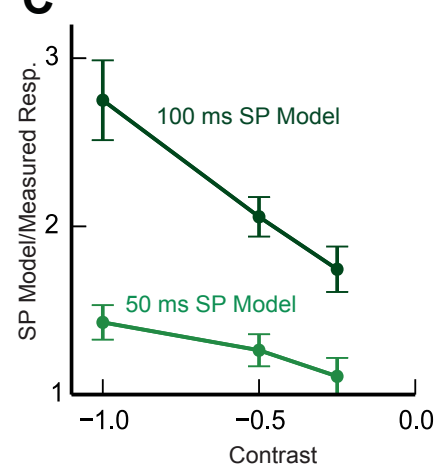

Figure 3. Linear superposition analysis reveals saturation of the OFF response. A. The sum of two or four time-shifted responses to a $25 \mathrm{~ms}$ flash (green) were compared with the measured response to a $50 \mathrm{~ms}$ and $100 \mathrm{~ms}$ flash, respectively (blue). Light shading represents standard deviation (5 trials). B. The average measured OFF response (peak outward current, normalized) to $100 \mathrm{~ms}$ flashes plotted against the predicted response from the superposition of 25 and $50 \mathrm{~ms}$ flashes for $25 \%$, $50 \%$ and $100 \%$ contrast (shades of blue). C. The population average ratio of peak superposition model OFF response to measured OFF response for 50 $\mathrm{ms}$ and $100 \mathrm{~ms}$ superposition models $(n=7)$. Error bars represent SEM.

The results from Figures 1-3 demonstrate rectification of inward and outward currents at the input to ON bipolar cells. This seems to contradict the linearity predicted by the $\mathrm{LN}$ models constructed in previous studies. In [28], for example, the transformation of light to salamander ON bipolar membrane voltage was characterized using low contrast $(30 \%)$ white noise stimulation. The nonlinearity in this model was measured by directly comparing the predicted membrane response to measured voltage response at the ON bipolar soma. This function was found to be essentially linear. This result is consistent with our finding that there is little to no rectification at lower contrasts (Figure 2A, C). We sought to verify that the nonlinear cells we recorded were truly linear at lower contrast. We presented interleaved unique and repeated $5 \mathrm{sec}$ sequences of binary white noise at $33 \%$ contrast and measured the postsynaptic current to construct an LN model. The linear filter 
(Figure 4A) and nonlinear function (Figure 4B) were calculated from the responses to the unique sequences as described previously in [28, 35]. The linear filter was biphasic, with a transient inward component around $100 \mathrm{~ms}$, and a broader outward component between 150 and $300 \mathrm{~ms}$. The measured nonlinearity approximated a straight line and did not saturate, demonstrating a lack of rectification at $33 \%$ contrast. This $L N$ model was then applied to the repeated stimulus sequence and compared to the average measured response (Figure $4 \mathrm{C}$ ). While the model does not exactly match the measured response, the normalized root mean squared error between model and measured current was 18.1 $\pm 2.0 \%$ ( \pm SEM,$n=7$ ), indicating that the model accounted for more than $80 \%$ of the structure of the light response.
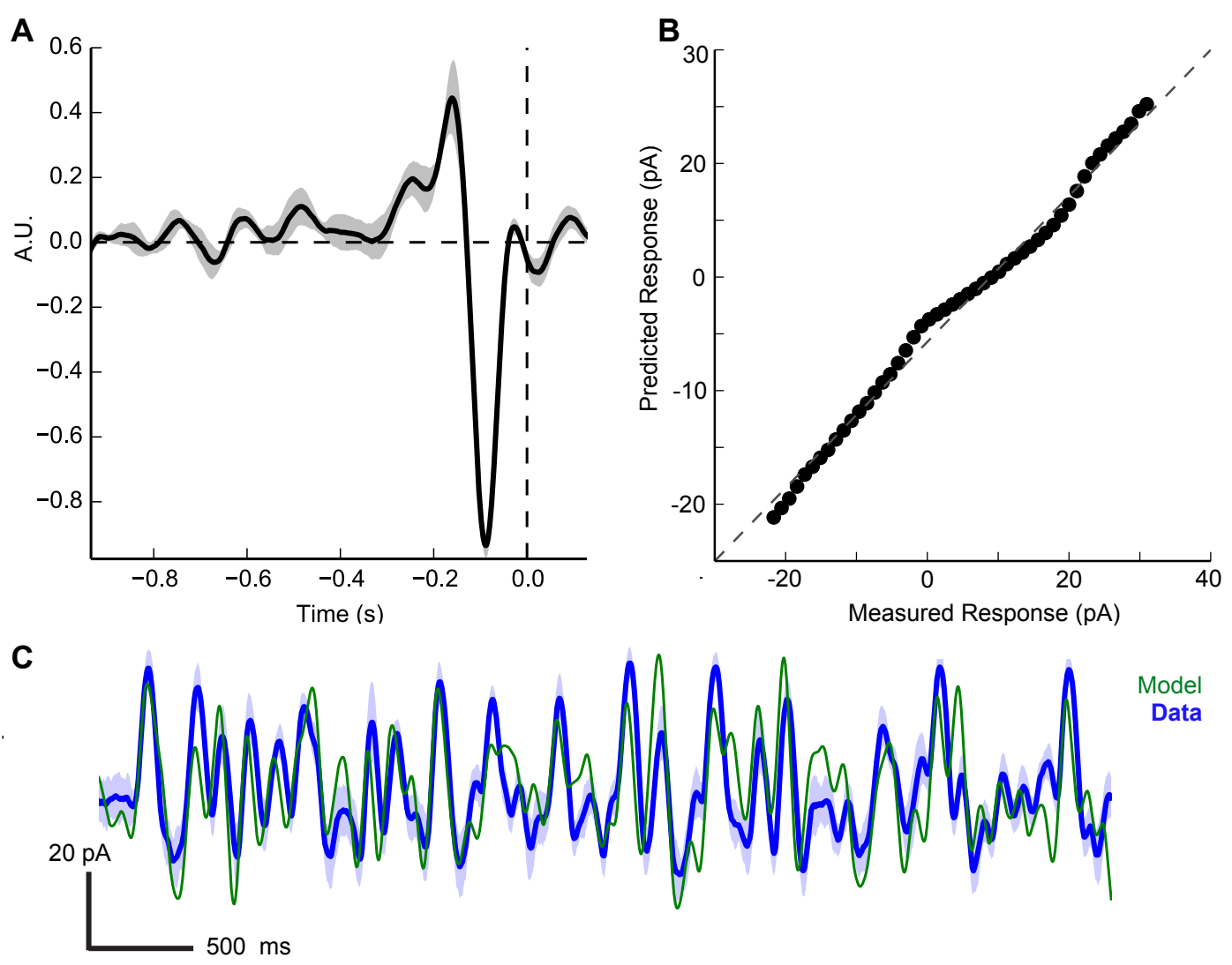

Figure 4. LN model of ON bipolar cell current. A. Linear filter of a voltage-clamped ON bipolar cell stimulated with $33 \%$ contrast binary white noise. B. Static nonlinearity calculated by comparing the measured response with the convolution of the stimulus and linear filter. C. LN model prediction (green trace) vs. average measured current (blue trace). Light blue trace represents standard deviation (5 trials).

The LN model assumes that both the linear filter and nonlinearity are time-invariant. To test this assumption, we presented paired 50 ms flashes at $100 \%$ contrast while varying the inter-flash interval (IFI) from 0 to $400 \mathrm{~ms}$ (Figure 5A). The principle of linear superposition predicts that the measured response should equal the sum of the response to two individual flashes with the proper time delay. A cell that exhibits history dependence, 
on the other hand, would fail this test for linear superposition because the first flash would change the response sensitivity of the cell for the second flash.

A

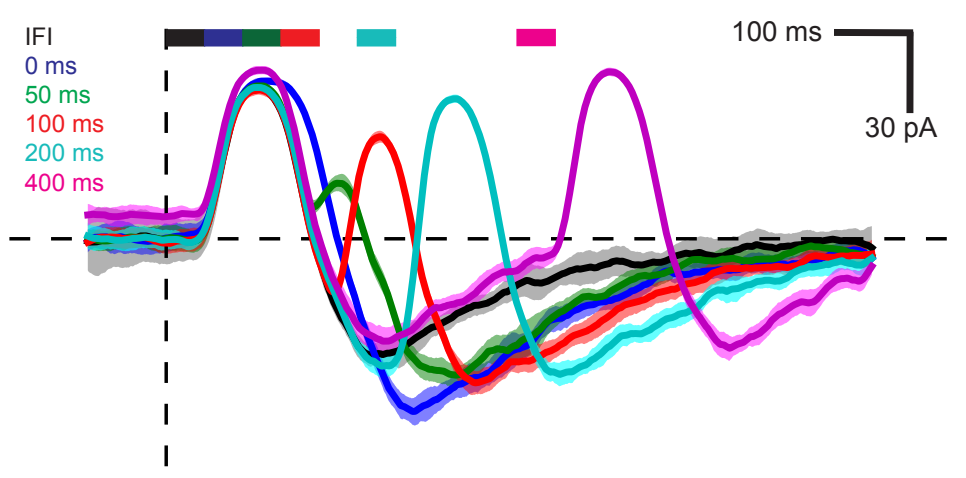

B
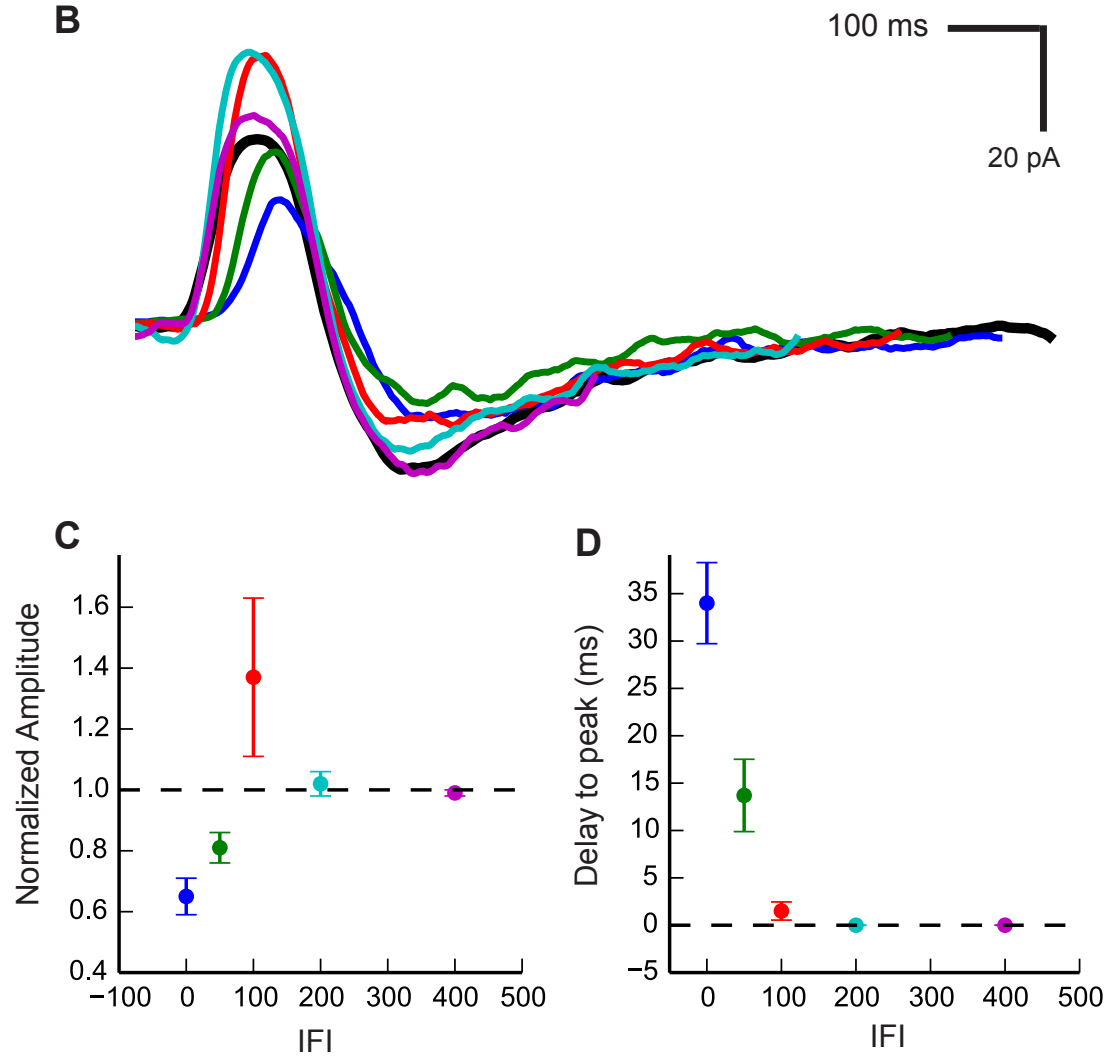

Figure 5. Paired high contrast flashes reveal gain control in ON bipolar cells. A. Responses to paired flashes with varying inter flash intervals (IFI) plotted against a single flash (black) for reference. Shaded regions around each trace represent standard deviation. Each flash was $50 \mathrm{~ms}$ in duration and $100 \%$ contrast. Vertical and horizontal dashed lines represent time zero and zero current, respectively. B. Single flash response (black) compared with the residuals after subtracting the single flash from paired flashes for all IFIs. C. Normalized amplitude of peak outward current for all IFIs. Error bars indicate SEM ( $n=9)$. D. Time delay to peak outward current for all IFIs. Error bars indicate SEM $(n=9)$.

To test this prediction, we examined the residual after subtracting the single flash from the 
response to the paired flash, as shown in Figure 5B. The outward current exhibited a delay to peak for the $0 \mathrm{~ms}$ and $50 \mathrm{~ms}$ IFI conditions (the former case is identical to a single $100 \mathrm{~ms}$ duration flash) as well as a modulation of the peak amplitude that varied as a function of IFI. Additionally, the amplitude of the outward current never exceeded the amplitude of the single flash, which is consistent with the saturation effect described in Figure 3. As shown in Figure $5 \mathrm{C}$, the reduction in peak amplitude was significant for $0 \mathrm{~ms}$ and $50 \mathrm{~ms} \mathrm{IFI} \mathrm{(} p=0.001$ and $p=0.014$, respectively, $n=9)$. The delay to peak, quantified for the population of cells in Figure 5D, was significant for the $0 \mathrm{~ms}$ and $50 \mathrm{~ms}$ IFI ( $p=0.0005$ and $p=0.019$, respectively). By $400 \mathrm{~ms}$, both the time course and amplitude of the second flash response matched the single flash response, indicating recovery to the baseline state before stimulation. This paired flash depression and subsequent recovery indicate the presence of a rapid, transient gain control at the input to ON bipolar cells.

In order to quantitatively describe the transient input nonlinearities present in ON bipolar input current, we constructed a phenomenological model of the light to ON bipolar current system. A block diagram of this model is shown in Figure 6A. The transformation from light to photoreceptor voltage can be modeled as a linear system, even at high contrasts [38]. To calculate the voltage response at the photoreceptor, $L(t)$, we convolved the light stimulus (in units of Weber contrast) with an alpha function that approximated the impulse response of the photoreceptor membrane. The amount of glutamate released at the cone pedicle as a function of membrane voltage goes to zero in response to very strong bright flashes. We approximate this asymmetric synaptic activation by passing the voltage response $L(t)$ through a static saturating nonlinearity $N_{1}(t)$ that amplifies the response to dark flashes and suppresses the response to dark flashes to yield the glutamate release, $u(t)$. This nonlinearity is similar to the synaptic activation described in [39], in which the resting voltage of the photoreceptor sits at the foot of the sigmoidal synaptic activation curve.

In the next stage of the model, we sought to capture the dynamics of the mGluR6 receptor to TRP channel system. The G-protein coupled signaling cascade in ON bipolar cells has not been fully characterized, ruling out the possibility of producing a biophysically realistic model. The LNK model published by [37] can accurately recapitulate the rapid transient voltage response of ganglion cells at transitions from low to high contrast by incorporating first-order rate dynamics. By adding a similar kinetics block in our model, we can simplify the signaling elements of the mGluR6 second messenger cascade into three pools of components: a ready pool $R$ that determines the instantaneous gain of the system, an activated pool $A$ that gates the TRP channel, and an inactivated pool $X$ that must be converted back to the ready state to participate in signaling. This form of model produces gain control as well as an overshoot at stimulus offset, and does not depend on a particular biophysical mechanism. 


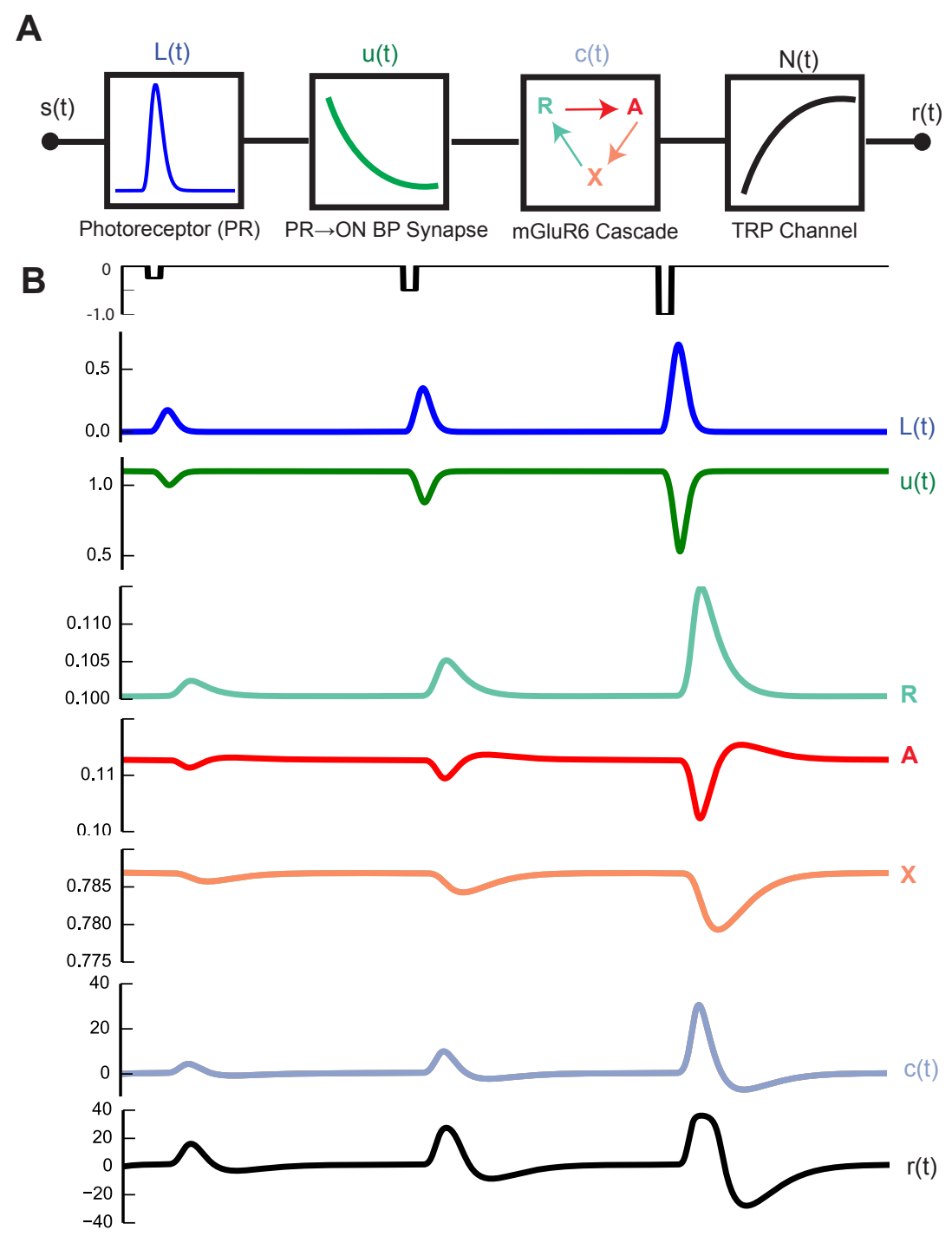

Figure 6. LNKN model of light to ON bipolar cell current. A. Block diagram of the model. B. Dynamics of each internal state variable of the LNKN model during responses to dark flashes of three different contrasts.

The maximum outward current of the system saturates at high contrast and long duration (see Figure 3). To model this second rectification, the current response $I(t)$ is calculated by passing the output of the kinetic block $A(t)$ through a saturating nonlinearity $N_{2}(t)$ that limits the maximum amplitude and scales the response to units of $p A$. The internal variables of this model are shown for dark flashes of increasing contrast in Figure 6B.

This linear-nonlinear-kinetic-nonlinear (LNKN) model successfully captures many of the key features of the ON bipolar response. In response to $33 \%$ white noise, the normalized root mean squared error between model and data is $13.4 \%$, compared to $18 \%$ for the $\mathrm{LN}$ model (Figure 7A vs. Figure 4). The response to a single flash is biphasic, with a significant 
overshoot at higher contrasts. The maximal inward current and outward current both saturate at higher contrast, and the amplitude of the response to dark flashes is larger than the response to a bright flash of equal contrast (Figure 7B and 7C), recapitulating the asymmetry shown in Figure 1 and the rectification described in Figures 2 and 3.

A

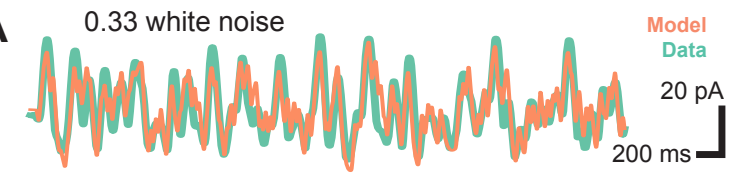

B Dark Flashes
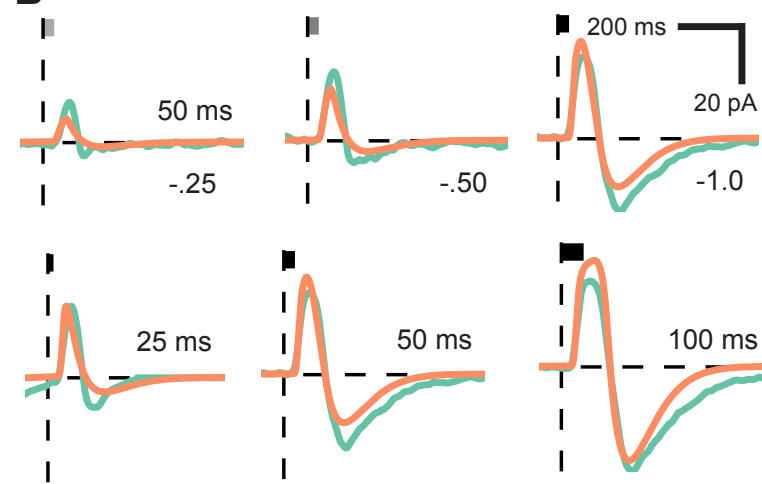

C Bright Flashes
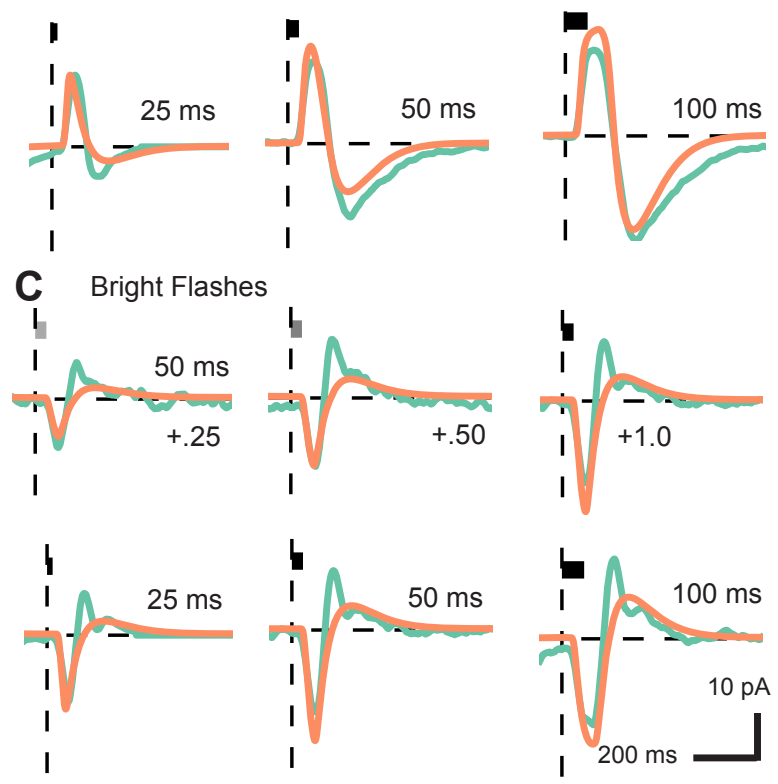

D Paired Dark Flashes
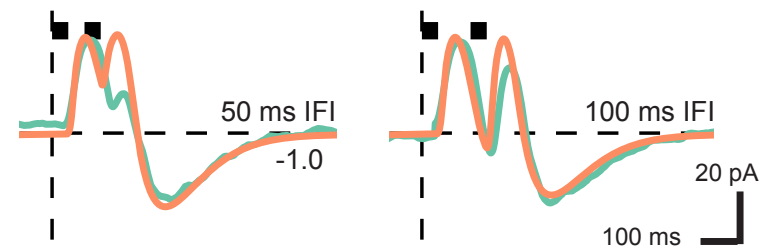

Figure 7. The LNKN model captures the key features of ON bipolar cell responses. A. Model (orange) and data (green) in response to $33 \%$ white noise. B. Model (orange) and data (green) in response to dark flashes of 25, 50 and 100 ms duration and dark flashes of $25 \%, \quad 50 \%$ and $100 \%$ contrast. C. Model (orange) and data (green) in response to bright flashes of 25, 50 and $100 \mathrm{~ms}$ duration and bright flashes of $25 \%, 50 \%$ and $100 \%$ contrast. D. Model (orange) and data (green) in response to paired flashes with $50 \mathrm{~ms}$ and $100 \mathrm{~ms}$ inter-flash interval.

A key feature of the model is the emergence of the large overshoot at $-100 \%$ contrast. The rate of $X \rightarrow R$ is $\sim 10 \mathrm{x}$ slower than the rate of $R \rightarrow A$ and $A \rightarrow X$. At stimulus offset, the inactive pool $X$ remains higher than baseline, reducing the ready pool $R$ such that the activation $A$ is reduced below the baseline level. This produces the overshoot at the offset. Before the final rectification stage $N_{2}(t)$, the amplitude of the peak model response to a $100 \%$ flash is approximately twice the response to a $50 \%$ contrast flash. The rectification suppresses 
the amplitude of the outward component while preserving the large inward overshoot generated by the kinetic block.

\section{Discussion}

Our study describes three distinct types of nonlinearity in ON bipolar cells. The first is the asymmetry between the response to bright and dark flashes at high contrast due to the suppression of the bright flash response (Figure 1). The second is the saturation of the OFF response revealed by the failure of the linear superposition model (Figure 3). The third is the rapid, transient paired flash depression (Figure 5).

\section{Mechanisms of rectification}

At low contrast, ON bipolar cells operate in the linear regime, with symmetric responses to increments and decrements. However, at high contrast, both bright and dark flashes appear to exceed the dynamic range of the mGluR6 pathway (Figure 2). Fahey and Burkhardt reported that in $\mathrm{ON}$ bipolar cells, the maximum amplitude of the voltage response to negative contrast steps grew as a function of background intensity, while the response to positive steps remained constant [40]. The asymmetry observed in Figure 1, in which the OFF response of ON bipolar cells is significantly larger than the ON response, was measured with a background intensity well into the photopic range, and is thus consistent with these findings. Furthermore, our measurements were performed in voltage-clamp, which eliminates the transformation from synaptic current to membrane voltage as a source of this asymmetry. One plausible mechanism is that a sufficiently intense bright flash hyperpolarizes cone photoreceptors to the point where they no longer release glutamate, limiting the amplitude of the inward current produced. In our model, this rectification is modeled by the synaptic activation function $u(t)$. Alternatively, a strong bright flash could sufficiently deactivate the mGluR6 pathway to open all of the available TRPM channels, limiting the maximal conductance.

The saturation of the OFF response to longer duration flashes (Figure 3), on the other hand, could result from a number of possible mechanisms. At high contrast, all of the readily releasable pool at the cone ribbon synapse could be released at once. Alternatively, the flooding of the synaptic cleft with glutamate could saturate all available mGluR6 receptors. A third possibility is that sufficiently strong glutamate input could drive the mGluR6 cascade to close all of the available TRPM channels. All three mechanisms ultimately limit the maximum amplitude of the response and are captured by the $N_{1}(t)$ rectification in the model.

\section{Mechanisms of rapid gain control}

The LN models calculated to study contrast adaptation in ON bipolar cells [28] and the 
transfer functions calculated with sinusoidal stimulation [41] represent the steady-state response properties of the cell. However, these models were constructed by explicitly excluding transient responses at the start of the sequence, limiting their utility in predicting the response to transient stimuli. The ON bipolar cells recorded in this study were approximately linear at steady-state and low contrast stimulation (Figure 4), but exhibited a time-dependent modulation of gain in response to $100 \%$ contrast dark flash stimuli (Figure 5A). Sharp gain reduction occurred within $50 \mathrm{~ms}$ of the first flash, ruling out slower gain control mechanisms such as the adaptation to mean luminance, which has a timescale of seconds, and calcium-mediated desensitization of the mGluR6 cascade [25], which has a timescale of hundreds of milliseconds. Furthermore, the reduction in gain occurred under voltage-clamp, ruling out the possibility of negative feedback via voltagegated ion channels.

One possible mechanism is feedback from amacrine cells onto the bipolar cell terminals. Bipolar cells are electrotonically compact, so inhibitory input at the axon terminal forms a component of the measured current. Another possibility is that the gain reduction is mediated by negative feedback within the mGluR6 transduction cascade. In addition to intracellular calcium, there are many elements of the second messenger cascade that could contribute to the temporal response properties observed in the input current. For example, in rod bipolar cells, glutamate binds to mGluR6, activating the alpha subunit of the G-protein, which in turn triggers DAG $\rightarrow P L C \rightarrow P K C \alpha$. The activation of PKCa has been shown to potentiate the current through the TRPM1 channel [42]. While this exact pathway does not exist in $\mathrm{ON}$ cone bipolar cells, perhaps there is a modulatory signaling element within the mGluR6 cascade that can modulate the gain further than the $20 \%$ gain reduction explained by the 3-state kinetic block.

\section{Limitations of the model}

While the model successfully captures many of the salient response features of the measured input current, it does not capture the rapid and transient outward current following bright flashes (Figure 7C). One possibility is that this outward current is caused by inhibitory feedback from an amacrine cell. Such a process would be completely outside of the scope of the model we have formulated. In addition, the 3-state kinetics block can only produce a $\sim 20 \%$ reduction in gain. This gain reduction is not sufficient to match the $>20 \%$ gain reduction of a paired second flash that occurs $50 \mathrm{~ms}$ after the first flash. The model accurately predicts the response for the $100 \mathrm{~ms}$ inter-flash interval (Figure 7D). In general, our model aims to achieve a balance between accuracy and simplicity. Simplicity is valuable because the resulting model has relatively few parameters that can be constrained by existing data. As further information becomes available about the biochemical cascade initiated by the activation of the mGluR6 receptor as well as about the types of amacrine cells that provide input to ON bipolar cells, further refinements of this form of model will likely become possible. 


\section{Implications for visual coding}

In general, rectification and gain control mechanisms serve to compress the signals to maximize efficient use of the dynamic range of neuronal output. While the distribution of contrasts in natural scenes is heavily weighted towards small contrasts and low intensities, the distribution has a long tail of high contrast stimuli [43-46]. Thus, an important operation for the retina to carry out is to compress this high dynamic range of light intensities into the limited dynamic range of neural signaling. We observed significant nonlinearities in the input current to ON bipolar cells starting at Weber contrasts of $\pm 50 \%$. Given the wide range of light intensities found in natural scenes, we expect that this nonlinear signaling regime will be achieved often. We also observed that nonlinear signaling in ON bipolars was enhanced by changes in light intensity that persisted for 50 ms or longer. Naturalistic visual stimuli also exhibit a wide range of durations over which light intensity fluctuates [43], which again makes it likely that nonlinear signaling in $\mathrm{ON}$ bipolars will be engaged.

Another important feature of natural visual scenes is the overrepresentation of dark contrasts [47]. This property results from the skew of the distribution of light intensities and has been related to the fact that retinas of many species have a higher density of OFFtype retinal ganglion cells than ON-type [48-51]. In this vein, we observed a much higher gain for OFF-responses than for ON - both in the peak outward current and in the overshoot inward current. This higher gain is matched to the greater prevalence of dark contrasts and may help to better encode the spatial information contained in dark contrasts. 


\section{REFERENCES}

1. Euler, T., et al., Retinal bipolar cells: elementary building blocks of vision. Nat Rev Neurosci, 2014. 15(8): p. 507-19.

2. Demb, J.B., et al., Bipolar cells contribute to nonlinear spatial summation in the brisk-transient (Y) ganglion cell in mammalian retina. J Neurosci, 2001. 21(19): p. 7447-54.

3. Shapley, R.M. and J.D. Victor, Nonlinear spatial summation and the contrast gain control of cat retinal ganglion cells. J Physiol, 1979. 290(2): p. 141-61.

4. Victor, J.D. and R.M. Shapley, The nonlinear pathway of $Y$ ganglion cells in the cat retina. J Gen Physiol, 1979. 74(6): p. 671-89.

5. Schwartz, G.W., et al., The spatial structure of a nonlinear receptive field. Nat Neurosci, 2012. 15(11): p. 1572-80.

6. Barlow, H.B. and W.R. Levick, The mechanism of directionally selective units in rabbit's retina. J Physiol, 1965. 178(3): p. 477-504.

7. Olveczky, B.P., S.A. Baccus, and M. Meister, Segregation of object and background motion in the retina. Nature, 2003. 423(6938): p. 401-8.

8. Chen, E.Y., et al., Alert response to motion onset in the retina. J Neurosci, 2013. 33(1): p. 120-32.

9. Singer, J.H. and J.S. Diamond, Sustained Ca2+ entry elicits transient postsynaptic currents at a retinal ribbon synapse. J Neurosci, 2003. 23(34): p. 10923-33.

10. Burrone, J. and L. Lagnado, Synaptic depression and the kinetics of exocytosis in retinal bipolar cells. Journal of Neuroscience, 2000. 20(2): p. 568-578.

11. Neves, G. and L. Lagnado, The kinetics of exocytosis and endocytosis in the synaptic terminal of goldfish retinal bipolar cells. J Physiol, 1999. 515 ( Pt 1): p. 181-202.

12. Chen, E.Y., et al., The neural circuit mechanisms underlying the retinal response to motion reversal. Journal of Neuroscience, 2014. in press.

13. Gjorgjieva, J., H. Sompolinsky, and M. Meister, Benefits of pathway splitting in sensory coding. J Neurosci, 2014. 34(36): p. 12127-44.

14. DeVries, S.H. and E.A. Schwartz, Kainate receptors mediate synaptic transmission between cones and 'Off' bipolar cells in a mammalian retina. Nature, 1999. 397(6715): p. 157-60.

15. DeVries, S.H., Bipolar cells use kainate and AMPA receptors to filter visual information into separate channels. Neuron, 2000. 28(3): p. 847-856.

16. Awatramani, G.B. and M.M. Slaughter, Intensity-dependent, rapid activation of presynaptic metabotropic glutamate receptors at a central synapse. J Neurosci, 2001. 21(2): p. 741-9.

17. Shen, Y., et al., A transient receptor potential-like channel mediates synaptic transmission in rod bipolar cells. J Neurosci, 2009. 29(19): p. 6088-93.

18. Koike, C., et al., TRPM1 is a component of the retinal ON bipolar cell transduction channel in the mGluR6 cascade. Proc Natl Acad Sci U S A, 2010. 107(1): p. 332-7.

19. Morgans, C.W., R.L. Brown, and R.M. Duvoisin, TRPM1: the endpoint of the mGluR6 signal transduction cascade in retinal ON-bipolar cells. Bioessays, 2010. 32(7): p. 609-14.

20. $\mathrm{Xu}, \mathrm{Y}$., et al., mGluR6 deletion renders the TRPM1 channel in retina inactive. J Neurophysiol, 2012. 107(3): p. 948-57. 
21. Field, G.D. and F. Rieke, Nonlinear signal transfer from mouse rods to bipolar cells and implications for visual sensitivity. Neuron, 2002. 34(5): p. 773-85.

22. Sampath, A.P. and F. Rieke, Selective transmission of single photon responses by saturation at the rod-to-rod bipolar synapse. Neuron, 2004. 41(3): p. 431-43.

23. Burkhardt, D.A., P.K. Fahey, and M. Sikora, Responses of ganglion cells to contrast steps in the light-adapted retina of the tiger salamander. Vis Neurosci, 1998. 15(2): p. 219-29.

24. Wu, S.M., F. Gao, and B.R. Maple, Functional architecture of synapses in the inner retina: segregation of visual signals by stratification of bipolar cell axon terminals. J Neurosci, 2000. 20(12): p. 4462-70.

25. Nawy, S., Desensitization of the mGluR6 transduction current in tiger salamander On bipolar cells. J Physiol, 2004. 558(Pt 1): p. 137-46.

26. Snellman, J., et al., Regulation of ON bipolar cell activity. Prog Retin Eye Res, 2008. 27(4): p. 450-63.

27. Curlander, J.C. and V.Z. Marmarelis, A linear spatio-temporal model of the lightto-bipolar cell system and its response characteristics to moving bars. Biol Cybern, 1987. 57(6): p. 357-63.

28. Rieke, F., Temporal contrast adaptation in salamander bipolar cells. Journal of Neuroscience, 2001. 21(23): p. 9445-9454.

29. Baccus, S.A. and M. Meister, Fast and slow contrast adaptation in retinal circuitry. Neuron, 2002. 36(5): p. 909-19.

30. Baccus, S.A., et al., A retinal circuit that computes object motion. J Neurosci, 2008. 28(27): p. 6807-17.

31. Burkhardt, D.A., P.K. Fahey, and M.A. Sikora, Retinal bipolar cells: temporal filtering of signals from cone photoreceptors. Vis Neurosci, 2007. 24(6): p. 76574.

32. Mao, B.Q., P.R. MacLeish, and J.D. Victor, The intrinsic dynamics of retinal bipolar cells isolated from tiger salamander. Vis Neurosci, 1998. 15(3): p. 425-38.

33. Makino, C.L., W.R. Taylor, and D.A. Baylor, Rapid charge movements and photosensitivity of visual pigments in salamander rods and cones. J Physiol, 1991. 442: p. 761-80.

34. Ma, J., et al., A visual pigment expressed in both rod and cone photoreceptors. Neuron, 2001. 32(3): p. 451-61.

35. Jarsky, T., et al., A Synaptic Mechanism for Retinal Adaptation to Luminance and Contrast. Journal of Neuroscience, 2011. 31(30): p. 11003-11015.

36. Kim, K.J. and F. Rieke, Temporal contrast adaptation in the input and output signals of salamander retinal ganglion cells. Journal of Neuroscience, 2001. 21(1): p. 287-299.

37. Ozuysal, Y. and S.A. Baccus, Linking the Computational Structure of Variance Adaptation to Biophysical Mechanisms. Neuron, 2012. 73(5): p. 1002-1015.

38. Clark, D.A., et al., Dynamical adaptation in photoreceptors. PLoS Comput Biol, 2013. 9(11): p. e1003289.

39. van Hateren, H., A cellular and molecular model of response kinetics and adaptation in primate cones and horizontal cells. J Vis, 2005. 5(4): p. 331-47.

40. Fahey, P.K. and D.A. Burkhardt, Effects of light adaptation on contrast processing in bipolar cells in the retina. Vis Neurosci, 2001. 18(4): p. 581-97.

41. Burkhardt, D.A., T.M. Bartoletti, and W.B. Thoreson, Center/surround organization of retinal bipolar cells: High correlation of fundamental responses of center and surround to sinusoidal contrasts. Vis Neurosci, 2011. 28(3): p. 18392. 


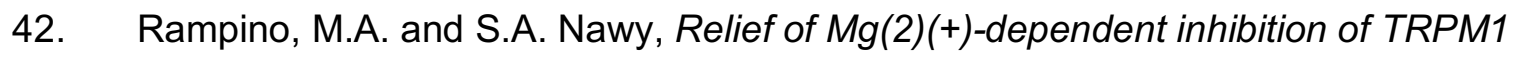
by PKCalpha at the rod bipolar cell synapse. J Neurosci, 2011. 31(38): p. 13596603.

43. van Hateren, J.H., Processing of natural time series of intensities by the visual system of the blowfly. Vision Res, 1997. 37(23): p. 3407-16.

44. Burkhardt, D.A., P.K. Fahey, and M.A. Sikora, Natural images and contrast encoding in bipolar cells in the retina of the land-and aquatic-phase tiger salamander. Vis Neurosci, 2006. 23(1): p. 35-47.

45. Tkacik, G., et al., Natural images from the birthplace of the human eye. PLoS One, 2011. 6(6): p. e20409.

46. Ruderman, D.L. and W. Bialek, Statistics of natural images: Scaling in the woods. Phys Rev Lett, 1994. 73(6): p. 814-817.

47. Ratliff, C.P., et al., Retina is structured to process an excess of darkness in natural scenes. Proc Natl Acad Sci U S A, 2010. 107(40): p. 17368-73.

48. Dacey, D.M. and M.R. Petersen, Dendritic field size and morphology of midget and parasol ganglion cells of the human retina. Proc Natl Acad Sci U S A, 1992. 89(20): p. 9666-70.

49. Morigiwa, K., M. Tauchi, and Y. Fukuda, Fractal analysis of ganglion cell dendritic branching patterns of the rat and cat retinae. Neurosci Res Suppl, 1989. 10: p. S131-9.

50. Wassle, H., L. Peichl, and B.B. Boycott, Morphology and topography of on- and off-alpha cells in the cat retina. Proc R Soc Lond B Biol Sci, 1981. 212(1187): p. 157-75.

51. Devries, S.H. and D.A. Baylor, Mosaic arrangement of ganglion cell receptive fields in rabbit retina. J Neurophysiol, 1997. 78(4): p. 2048-60. 